\title{
HISTOIRE ÉCONOMIQUE ET SOCIALE
}

Jacques LE Goff, La Bourse et la vie :économie et religion au Moyen Âge. Paris, Hachette, 1986. $11 \times 18,126$ p. (« Textes du xx siècle »).

Dans ce bref essai, Jacques Le Goff étudie le statut réservé par l'Église à l'usure et aux usuriers, question centrale dans bien des réflexions éthiques et religieuses du Moyen Âge. Le XIII siècle est au centre de cet ouvrage car, d'une part, les valeurs traditionnelles de l'idéal chrétien se trouvent menacées par la diffusion de l'économie monétaire et l'essor de l'usure ; d'autre part, l'Église, confrontée à la montée des hérésies et face à une société en pleine mutation, "choisit de parler » (p. 15), ce qui induit une renaissance de la prédication. De ce fait, la source principale de l'auteur est constituée par les exempla (exemples donnés comme véridiques et insérés surtout dans des sermons), auxquels il faut ajouter les traités théologiques et les manuels de confesseur rendus nombreux par l'essor de la confession après 1215 et le IVe concile du Latran.

Dans le deuxième chapitre, J. Le Goff présente le cadre théorique de son analyse, qu'il situe dans la perspective développée par Karl Polanyi : d'une part, la notion de réciprocité domine les échanges économiques ; d'autre part, l'économie se trouve encastrée dans des situations qui ne sont pas elles-mêmes de nature économique, ce qui justifie le recours à une analyse institutionnelle et conduit aux notions scolastiques de " juste prix " et de " juste salaire ".

L'argument majeur de l'Église contre l'usurier est que celui-ci vend le temps qui s'écoule entre le moment où il prête et celui où il est remboursé ; or le temps n'appartient qu'à Dieu (p. 42). Cette accusation est très souvent reprise par les clercs, d'autant plus sensibles à cette atteinte au monopole divin que la fin $\mathrm{du}_{\mathrm{XII}}{ }^{\mathrm{e}}$ et le XIII ${ }^{\mathrm{e}}$ siècle voient se multiplier ce type d'empiètement sur le domaine sacré. Dans le même ordre d'idées, l'auteur souligne l'accusation portée contre les intellectuels non clercs qui vivent de la vente de la science qui, comme le temps, n'appartient qu'à Dieu. À l'inverse, la valeur nouvelle du xilı ${ }^{\mathrm{e}}$ siècle est le travail, ce qui condamne l'usurier oisif qui s'enrichit sans travailler. Ce dernier agit donc contre le plan du Créateur, ce que souligne Thomas de Chobham : "L'usurier veut acquérir un profit sans aucun travail et même en dormant, ce qui va contre le précepte du Seigneur qui dit : “ À la sueur de ton visage, tu mangeras ton pain " " (p. 45). Son salut est donc en question car si les excuses se multiplient au xiıl siècle pour nombre de métiers jusqu'alors illicites, l'usurier reste avec les prostituées et les jongleurs condamné « en soi » (p. 53). J. Le Goff cite à ce propos un sermon original de J. de Vitry qui présente une variante de la société trifonctionnelle médiévale : «Dieu a ordonné trois genres d'hommes, les paysans et autres travailleurs pour assurer la subsis- 
tance des autres, les chevaliers pour les défendre, les clercs pour les gouverner, mais le diable en a ordonné une quatrième, les usuriers. Ils ne participent pas au travail des hommes et ils ne seront pas châtiés avec les hommes mais avec les démons. Car à la quantité d'argent qu'ils reçoivent de l'usure correspond la quantité de bois envoyée en enfer pour les brûler " (p. 61). L'auteur souligne combien ce dédoublement de la troisième fonction manifeste la méfiance des intellectuels à l'égard de la sphère des échanges qui se multiplient au XIII' siècle mais sans être assimilés à une activité économique fondée sur le travail comme l'agriculture.

À ce choix imposé à l'usurier : " la bourse ou la vie " vont progressivement se substituer des accommodements pour aboutir à une formule plus conciliante : " la bourse et la vie ". L'intégration de l'usurier à la société médiévale a été facilitée par deux éléments : la modération des taux pratiqués et l'existence d'un taux de marché accepté comme normal s'il reste à l'intérieur de certaines bornes ; l'émergence de nouvelles valeurs qui permet à la tradition scolastique de multiplier les "excuses", comme les notions d'indemnité ou d'incertitude. Plus fondamentalement, c'est l'apparition de l'idée de purgatoire qui a permis cette mutation. J. Le Goff en a ailleurs évoqué la naissance, il en rappelle ici brièvement les conditions. Nombre d'exempla étudient les exigences requises pour que l'usurier et ses proches puissent accéder au purgatoire. Puisque la condamnation définitive n'est plus la sentence unique prononcée par l'Église, la contrition, le repentir et la pénitence (la restitution des biens injustement acquis) à la veille de la mort constituent des moments essentiels dans la vie religieuse de l'usurier qui retrouve ainsi un certain libre arbitre face à sa vie ou sa mort éternelles. L'auteur voit dans cet espoir de salut un élément important dans l'évolution du système économique médiéval. Les initiateurs du capitalisme sont les usuriers, vendeurs de temps et d'argent, deux notions bien propres, notons-le, à définir le capital. Or, ce qui retient ces hommes au seuil du capitalisme, ce ne sont pas les condamnations terrestres de l'usure mais « la peur angoissante de l'enfer » (p. 99). De ce fait, "l'espoir d'échapper à l'enfer grâce au purgatoire permit à l'usurier de faire avancer l'économie et la société du xuIe siècle vers le capitalisme" ".

Ce beau livre présente de multiples attraits. Outre un recueil de textes médiévaux, souvent peu accessibles, consacrés au problème de l'usure, il offre une réflexion sur les formes que peut avoir la prise en compte des réalités économiques dans une société préindustrielle. Si, dans un premier temps, le seul outil culturel disponible est religieux, l'émergence d'une forme de pensée plus laïque, et la pression du milieu social obligent dans un deuxième temps le discours de l'Église à s'adapter. De ce fait, en dehors de ses apports propres à la réflexion historique sur le Moyen Âge, cet essai constitue une contribution à l'étude théorique des systèmes économiques sous l'angle des blocages mentaux que ceux-ci rencontrent et de leur dépassement. Cette voie de recherche, qui renoue avec de célèbres exemples de l'historiographie, peut certainement apporter beaucoup, comme J. Le Goff vient de nous le démontrer.

Jean-Yves GrenIER. 
George HUPPERT, After the Black Death. A Social History of Early Modern Europe. Bloomington/Indianapolis, Indiana University Press, 1986. $15,2 \times 23,4$, XVIII-171 p. (« MB », 404).

L'auteur, spécialiste américain du $\mathrm{xvI}^{\mathrm{e}}$ siècle européen et plus spécialement français, déclare, dans sa Préface, avoir voulu rédiger " une introduction à l'histoire sociale de l'Europe occidentale du XIve au Xvile siècle " (an introduction to the social history of Western Europe), de la peste bubonique de 1347 à sa dernière « apparition menaçante " (threatening appearance) à Marseille en $1721^{\prime}$. Pour y parvenir, il se réfère à l'approche historiographique des Annales et prétend s'appuyer sur des études de cas (case studies). Il s'affirme d'ailleurs conscient des pièges que recèle toute généralisation à partir d'études limitées dans le temps et surtout dans l'espace, et ne veut présenter que des conclusions hypothétiques (tentative).

Malgré quelques descriptions bien venues et des remarques parfois pénétrantes, l'auteur ne me paraît pas avoir réussi dans son entreprise. Il n'y aurait pas trop lieu de s'attarder sur les raisons de cet échec si celles-ci ne soulevaient pas des problèmes méthodologiques et conceptuels qui forment l'une des préoccupations de cette revue. D'abord, une introduction ne peut pas être une succession d'essais sur des thèmes singuliers plus ou moins bien reliés entre eux par des paragraphes de transition. Elle doit, au contraire, constituer une approche globale ; il lui faut repérer les zones d'ombre et de lumière, ce que l'on connaît et ce que l'on ignore, et le dire. Il convient également, pour chaque sujet abordé, de ne sélectionner le case study qu'en fonction des informations les plus larges possible, et de discuter à fond, en tout état de cause, les opinions divergentes. Il est curieux, par exemple, que les développements sur la noblesse anglaise se passent des recherches de Cliffe ou d'Everitt, que ceux sur celle de France se privent des analyses d'A. Jouanna et, plus stupéfiant encore, de collègues américains comme J. Dewald, J. L. Goldsmith ou J. B. Wood, qui auraient conduit $\mathrm{G}$. Huppert à nuancer sérieusement ses affirmations.

D'un autre côté, présenter la société rurale sans références aux synthèses de W. Abel, de Fr. Lütge et de G. Franz dans la Deutsche Agrargeschichte, des ouvrages collectifs de l'Histoire de la France rurale et de l'Agrarian History of England and Wales, apparaît aussi aberrant que de disserter sur la démographie sans citer l'école française, y compris J. Dupâquier et A. Perrenoud, pour ne pas parler des chercheurs italiens. Reléguer aux accessoires l'influence sur la société, des Réformes en général et du puritanisme en particulier (Ch. Hill n'aurait-il rien écrit ?) laisse un peu pantois, même si $G$. Huppert rappelle opportunément que la christianisation n'a pas toujours ni partout eu la profondeur qu'on lui attribue parfois. Une série d'essais autorise une certaine

1. p. IX. 
liberté ; une introduction qui oblige à une vue d'ensemble, certainement pas. Il y a ici confusion des genres.

Plus graves peut-être encore sont les simplifications abusives. Passons sur celles qui, comme à la page 75 , illustrent le mouvement des profits des paysans par celui des salaires agricoles et ceux-ci par celui du maçon (vivant, il est vrai, à la campagne). Oublions que P. Chaunu, dans Le Temps des Réformes n'entend pas par sécularisation de la société, une moindre emprise religieuse sur celle-ci, comme semble le croire l'auteur. Abordons les case studies: la guerre des paysans allemands n'est guère abordée que de Mühlhausen (deux pages), un coup de chapeau aux douze articles de Souabe mis à part (deux paragraphes). Ce qui inquiète ici, c'est que $G$. Huppert s'appuie sur des discussions " informelles" avec P. Blickle, R. Endres et W. Schultze et également sur le manuel que ceux-ci ont publié dans la collection U.T.B. ${ }^{2}$. Or dans leurs articles et dans leurs ouvrages, ces différents historiens donnent des événements de 1525 une vue plus large et proposent des interprétations plus complexes que celle avancée par notre auteur.

En fait, on touche là la faiblesse la plus grave de cette série d'essais : la faiblesse conceptuelle. Il n'y a aucune caractérisation des phénomènes évoqués, aucune Begriffsbildung systématique et explicite, consciente et volontaire. Or si une Begriffsbildung n'évite déjà pas les discussions (voir celles qu'entretiennent deux historiens allemands revendiqués par G. Huppert, P. Blickle et H. Schilling, sur une notion qui tombe en plein dans l'histoire sociale, celle de Gemeiner Mann), l'absence d'une telle démarche risque de conduire à bien des confusions d'autant plus sournoises et dangereuses qu'elles se montrent totalement inconscientes. Ce défaut de conceptualisation au niveau des phénomènes historiques se retrouve à celui des concepts fondamentaux. Ceux-ci tournent autour de la monocausalité, sorte de Deus ex machina. Ainsi, à en croire la conclusion, la conception de la propriété aurait été déterminante. Pareille recherche paraît un peu vaine car, à ce petit jeu, il est toujours possible d'opposer une cause première à une autre cause première ; un peu plus de sophistication serait sans doute non seulement souhaitable, mais utile.

Si l'attitude de G. Huppert ne soulevait pas autant de questions fondamentales, un bref compte rendu aurait suffi. Cependant, il ne faudrait pas déduire de sa longueur et des réserves qui s'y font jour, à une totale condamnation. Il y a à prendre dans ce livre, même s'il ne tient pas ses promesses. Surtout, tout historien commet des erreurs et il serait mal venu, après avoir critiqué ce livre, de jeter la pierre à son auteur.

Hugues NeveuX.

2. Horst Buszello, Peter Blickle, Rudolf Endres, eds, Der deutsche Bauernkrieg, Paderborn/Munich/Vienne/Zurich, Ferdinand Schöningh (« U.T.B.», 1275), 1984 p. 418 . 
Marie-Thérèse Boyer-Xambeu, Ghislain Deleplace, Lucien Gillard, Monnaie privée et pouvoir des princes. L'économie des relations monétaires à la Renaissance. Préf. de Pierre Jeannin. Paris, Presses de la Fondation nationale des sciences politiques/Ed. du C.N.R.S., 1986. $16 \times 24,423$ p.

La caractéristique première de cet ouvrage est son intention pluridisciplinaire. Trois économistes, aux préoccupations juqu'alors très contemporaines, entreprennent d'éclairer le mécanisme complexe des relations monétaires de la Renaissance, sujet jusqu'ici réservé à la sagacité et à l'érudition des historiens patentés. Défauts et réussites du livre résultent entièrement de cette donnée initiale. Dans un premier temps, les auteurs brossent le portrait des trois catégories d'agents qui sont impliqués dans les relations monétaires: les marchands-banquiers, les négociants ordinaires et les agents officiels. Cette partie, qui est à la fois la plus historique et la plus descriptive, est peu satisfaisante. D'une part, beaucoup d'aspects historiques très connus et souvent élémentaires sont trop longuement rappelés ; d'autre part, la typologie esquissée, en particulier celle des négociants, est critiquable et l'introduction des financiers dans cet ensemble monétaire peu justifiée. Dans un second temps, l'étude aborde plus spécifiquement les mécanismes créateurs de monnaie. À une présentation géographique de l'espace mondial du négoce succède le chapitre explicatif essentiel du livre (chapitre 5) consacré au mécanisme du change par lettre que les auteurs ont délibérément placé au centre de toutes leurs explications. Sa compréhension nécessite la mise en place de trois éléments abondamment décrits. En premier lieu, le commerce européen spécifique (et non mondial) exige une mobilité monétaire et donc l'existence d'un moyen de paiement transférable, la lettre de change. En second lieu, celle-ci peut exister grâce au seigneuriage prélevé par les princes lorsqu'ils battent monnaie. En effet, ce droit régalien assure une différence systématique entre les valeurs des monnaies nationales et étrangères en circulation, différence qui est à l'origine du gain obtenu par les marchands-banquiers lors de la vente de la lettre de change. Un des apports les plus importants de cet ouvrage est précisément d'expliquer les bénéfices des marchands-banquiers grâce à la création d'une véritable monnaie privée et non, comme le pensaient jusqu'alors les historiens à la suite de $\mathbf{R}$. de Roover, par l'intérêt perçu sur un prêt déguisé. La démonstration des auteurs est assez convaincante; on leur reprochera seulement de paraitre exclure que l'opération de change puisse revêtir différents aspects au profit de la seule systématisation monétaire. En troisième lieu, le change par lettre nécessite l'existence de foires internationales dans chaque pays qui autorisent la détermination périodique des cours des changes (le " conto ") et permettent d'opérer les transactions réelles. Les auteurs insistent beaucoup, tout au long du livre, sur le rôle particulier tenu par la "foire centrale " (Lyon puis "Bisenzone" après 1575-1580) sans laquelle la circulation européenne du change serait impossible. Cette hypothèse est assez séduisante en ce qu'elle laisse entrevoir un mécanisme centralisé, au fonctionnement à la fois harmo- 
nieux et rigoureux, qui tranche avec les autres processus économiques du $\mathrm{XVI}^{e}$ siècle que l'on peut connaître. Cependant, là encore, si les auteurs montrent de façon convaincante la possibilité d'un tel fonctionnement, ils ne démontrent ni sa nécessité ni sa réalité.

La troisième partie étudie les implications et la destinée de ce monnayage privé. La création de monnaie publique n'est pas neutre devant ce trafic de lettres de change puisque celles-ci ne s'expliquent que par la prérogative des princes à opérer des discriminations dans la valeur des monnaies en circulation. À l'inverse, la pratique du " conto " par les marchands-banquiers garantit indirectement l'autonomie des prérogatives monétaires royales. Il en résulte ainsi un "partage de la responsabilité sociale du monnayage " entre le public et le privé (p. 268). La pérennité de ce système repose cependant sur un ensemble de conditions tant économiques (les nécessités marchandes) que monétaires (une stabilité relative de la monnaie publique de la foire centrale, en l'occurrence la livre tournois). Or, à la fin du xvie siècle, de profondes transformations issues d'une conjoncture difficile détruisent la prééminence florentine et lyonnaise au profit des Gênois et des foires de " Bisenzone " (en fait surtout Plaisance après 1579). L'objet du chapitre 8 est de suivre ce déplacement géographique qui traduit le passage d'une fonction monétaire et économique du change à une fonction financière car les Gênois utilisent celui-ci pour assurer le financement des troupes espagnoles dans les Flandres. La désintégration du système lyonnais s'explique également par la célèbre ordonnance de 1577 qui supprime la monnaie de compte au profit du compte en écus, ce qui entraîne une quasi-suppression de la taxe de seigneuriage et donc de la source de profit des marchands-banquiers. Avec ce recentrage s'esquisse un mouvement national important de la fin du $\mathrm{xvI}^{\mathrm{e}}$ siècle et la disparition d'un mode original d'internationalisation de la monnaie. En d'autres termes, la « socialité monétaire " propre à la Renaissance que poursuivent les auteurs disparaît au cours des deux dernières décennies du Xvie siècle.

Le propos de ce livre - on l'aura compris - est donc de démontrer que l'essentiel de l'économie des relations monétaires de la Renaissance peut être analysé à travers le mécanisme de la lettre de change, articulé dans chaque État sur le monnayage public du prince. Il en résulte une attention centrée sur la seule technique monétaire, à l'exclusion de réflexions sur le système économique impliqué ou d'une contribution au grand débat sur la nature des sociétés monétaires. Or il ne va pas de soi qu'il est possible de comprendre les relations monétaires et les enrichissements qu'elles autorisent par le seul biais des techniques de création de la monnaie, surtout dans une société préindustrielle. À l'inverse, ce point de vue assure une grande homogénéité des explications et une rigueur dans la démonstration à laquelle peu d'ouvrages d'histoire peuvent prétendre. C'est ce dernier aspect qui fait la force de ce livre, au moins dans ses deuxième et troisième parties, avec la reconstruction d'un système monétaire autour de quelques mécanismes subtilement analysés avec un constant souci de cohérence. Les apports et les limites de cette lecture, la portée des interprétations proposées et les compléments à apporter sur certaines questions comme les mécanismes inflationnistes méritent un débat. Ce livre 
riche et dense pourrait ainsi être l'occasion d'une discussion entre économistes et historiens sur un thème négligé par ces derniers depuis trop longtemps.

Jean-Yves Grenier.

Antoin E. MURPHY, Richard Cantillon : Entrepreneur and Economist. Oxford, Clarendon Press, 1986. $16 \times 24,336$ p.

Antoin E. Murphy nous propose quelques aperçus très nouveaux sur la vie et l'œuvre du grand économiste irlandais Richard Cantillon, auteur du célèbre Essai sur la nature du commerce en général (1755). Ne cherchons pas dans son ouvrage une biographie traditionnelle. Si l'ordre chronologique nous permet de suivre l'économiste de ses débuts comme homme d'affaires jusqu'à son mystérieux décès, l'auteur préfère se consacrer à l'étude de certains aspects de sa personnalité, centrés autour de la figure du manieur d'argent. L'un des attraits du livre est la tentative d'éclairer l'œuvre de l'économiste par les expériences du banquier.

Le chapitre 4 nous décrit les débuts de la carrière de Cantillon et l'importance qu'a eue pour sa formation de financier son cousin, le chevalier Richard Cantillon, et son protecteur le duc de Chandos, payeur général des troupes anglaises prodigieusement enrichi. C'est à son service qu'il apprend le maniement des finances durant les dernières années de la guerre de Succession d'Espagne. La rupture se produit quand il choisit alors de ne pas retourner à son service à Londres mais d'établir sa propre banque d'affaires à Paris, vrai départ dans la carrière. Durant toute sa vie, l'activité bancaire de Cantillon s'exercera autour du pôle parisien. Ses débuts français seront facilités par l'existence d'un milieu irlandais catholique immigré très actif dans les milieux financiers. Son introduction dans les cercles intellectuels de la Régence, comme le Club de l'Entresol, rendue difficile par son manque de noblesse, fut favorisée par l'amitié que lui porta Bolingbroke. C'est aussi du temps de cette rencontre que datent les premiers écrits économiques sur le commerce et le luxe (vers 1718). L'installation de sa banque fut un succès grâce à la confiance qu'il avait su susciter chez les financiers britanniques et au réseau de relations et de contacts parmi les commerçants irlandais installés dans les grands ports comme Nantes et Bordeaux que son cousin avait constitué. Rapidement, cependant, son intérêt se porta vers les activités de John Law.

Sur les 10 chapitres qui suivent (de 5 à 15), 9 sont consacrés aux relations de Cantillon avec le système de Law et ses conséquences multiples. A. E. Murphy va désormais s'attacher à exposer avec le maximum de précision les opérations financières et bancaires que l'économiste irlandais a montées. Celui-ci en tira non seulement une fortune personnelle considérable puisqu'il fut un de ces millionnaires enrichis par la Compagnie du Mississipi, mais aussi de nombreux contacts intellectuels, en particulier avec Law, Melon et Dutot. 
La première opération a lieu en 1719 avec l'achat d'actions de la Compagnie du Mississipi. Sa force fut de savoir réaliser à temps sa fortune grâce à une rapide perte de confiance dans le Système, dont il anticipe une chute imminente, qui l'incite à quitter la France pour l'Italie dès août 1719. Il acquit à cette occasion une grande réputation pour la prévision des marchés (en fait; les actions ont atteint leur cours maximal en 1720, près de 5 fois leur niveau d'août 1719 !) (chapitre 5). Sa méfiance reposait, d'une part, sur la croissance trop rapide du stock monétaire et sur l'échec de la colonisation en Louisiane dont il connaissait les difficultés grâce à l'expédition de son frère Bernard en 1719 , minutieusement relatée au chapitre 6 . La deuxième opération financière de Cantillon fut le prêt de sommes importantes à des spéculateurs anglais, en particulier Joseph Gage et Lady Mary Herbert, désireux en 1720 de disposer de fonds pour spéculer sur le système. Ces affaires sont l'occasion pour A. E. Murphy de dresser le portrait de ces spéculateurs anglais, souvent peu au fait de la finance parisienne et qui ont alors contracté des dettes considérables (chapitre 7). La troisième source d'enrichissement, décrite encore avec beaucoup de précisions, se situe à la deuxième époque du Système, en 1720. La spéculation de Cantillon s'opère sur les taux de change monétaire et repose, plus que les deux premières, sur sa remarquable capacité intellectuelle à comprendre l'incompatibilité fondamentale entre une forte expansion monétaire qui induit un déficit de la balance des paiements et le taux de change monétaire qui ne peut que s'effondrer. Le livre III de l'Essai n'est que l'explication théorique de ce mécanisme que l'économiste sut exploiter pour sa fortune personnelle (chapitre 8). Le chapitre 9 est le dernier à avoir trait directement aux spéculations de Cantillon en 1719-1720, à l'extérieur de la France. Il contient en particulier, aux pages 187-188, une intéressante conclusion sur ses capacités de " market operator" sur les grandes places européennes pendant ces années confuses du Système. La fin de l'ouvrage est consacrée, d'une part, aux conséquences de cet enrichissement sur la vie de Cantillon et, d'autre part, à une présentation de l'Essai et de sa publication. Plusieurs spéculateurs appauvris l'ont accusé d'avoir fait fortune grâce à des prêts usuraires et, jusqu'à sa mort, il est menacé de nombreux procès et fit même deux brefs séjours en prison (chapitres 10 à 13). Son décès mystérieux dans l'incendie de sa résidence londonienne en 1734 a dès lors beaucoup préoccupé l'auteur. Cet incendie était jusqu'ici considéré comme criminel mais A. E. Murphy démontre le peu de fondement d'une telle thèse et suggère que Cantillon avait lui-même organisé un faux décès pour pouvoir échapper à la poursuite de ses ennemis et fuir hors d'Europe (on retrouverait sa trace dans le Surinam !) (chapitre 14). Deux chapitres seulement sont consacrés à l'Essai en tant que tel. Le chapitre 13 en présente les thèmes principaux et montre de façon convaincante comment ils sont reliés à l'expérience de Law, pourtant jamais mentionnée. Le dernier chapitre étudie les conditions de la publication en 1755 et l'influence qu'il a exercée au milieu du XviIIe siècle sur le groupe de Vincent de Gournay autour des ouvrages édités par le libraire Pierre-André Guillyn (1715-1781). 
L'ouvrage de A. E. Murphy, on le voit, contient une grande variété de propos. À travers Cantillon, c'est le milieu des financiers internationaux et le Système de Law qui est décrit et analysé. En ce sens, plus qu'une biographie il s'agit bien d'un livre d'histoire qui contribue à éclairer le milieu des financiers du XvIII siècle. Cantillon cependant est aussi un économiste et le deuxième intérêt est de montrer, non pas de façon générale mais par une approche précise de ses spéculations, dont la reconstitution minutieuse est remarquable, comment sa pensée économique est le fruit d'une activité d'homme d'affaires, comme le seront après lui Ricardo et Keynes. On peut regretter à l'inverse que le milieu intellectuel fréquenté par Cantillon ne soit pas brossé avec plus de précisions et que le destin de l'Essai, son influence au-delà du milieu de Gournay des années 1760 , soient traités si rapidement. Ce n'était cependant pas le propos de l'auteur qui s'est consacré à l'entrepreneur et à l'économiste plus qu'au penseur inscrit dans une histoire de longue durée. Cet objectif, somme toute original, d'expliquer l'histoire de la pensée économique par son inscription dans la réalité vécue est atteint et de fort belle manière.

Jean-Yves GrenIER.

William N. Parker, Economic History and the Modern Economist. Oxford, Basil Blackwell, 1986. 14,4 × 22,2, 105 p.

Les relations entre l'histoire économique et la théorie économique n'ont jamais été simples et certainement pas aussi fructueuses qu'elles auraient dû l'être. La peur des historiens devant la théorie et la conceptualisation de cette science plus "dure " qu'est l'économie, la méconnaissance, voire le manque d'intérêt, des économistes pour les époques antérieures aux nôtres, expliquent cette absence de dialogue. C'est à éclairer celle-ci et à y remédier que s'emploie Economic History and the Modern Economist. Ce livre est le résultat d'une discussion entre économistes et historiens économistes américains qui eut lieu en 1984 sous l'égide de l'American Economic Association. Deux théoriciens de réputation mondiale, K. J. Arrow et $\mathrm{R}$. E. Solow, représentent le versant purement économique. Arrow assimile les liens de l'histoire économique et de l'économie à ceux de la géologie et de la physique : la première utilise pour expliquer des phénomènes précis des explications théoriques fournies par la seconde. Pour l'économiste de Stanford, il est ainsi évident que les approches de la théorie économique sont utiles à l'histoire comme le montre l'exemple célèbre des relations entre le chemin de fer et la croissance économique au $x x^{e}$ siècle. En sens inverse, l'histoire fournit à la science économique des preuves empiriques pour tester ses hypothèses et théories et, plus fondamentalement, elle détermine l'ampleur du conditionnement des phénomènes économiques par l'histoire. R. E. Solow est nettement plus pessimiste. Il pose d'emblée que «la théorie économique n'apprend rien de l'histoire économique " et que " celle-ci est plus corrompue qu'enrichie par celle-là » (p. 21). Il 
reprend l'image d'Arrow d'une science économique comme physique de la société mais pour critiquer l'universalité qu'elle prétend attribuer à un modèle unique de fonctionnement du monde alors qu'une pluralité d'hypothèses et d'interprétations sont nécessaires. L'apport de séries économiques par l'histoire est discutable car les tests économétriques exigent des séries très longues et une stationnarité que l'on trouve rarement, et en l'absence desquelles tout et son contraire sont démontrables. L'histoire économique n'est pas plus satisfaisante car ses travaux reposent le plus souvent sur une technicité qui remplace une pensée défaillante (p. 26). La science économique que Solow envisage ne repose pas sur un apport de l'histoire mais sur une fusion des deux disciplines. Le théoricien formulera alors une pluralité de modèles dont les contraintes réelles et institutionnelles seront déterminées par la réflexion historique. Les possibilités multiples de fonctionnement du marché du travail constituent (pour l'auteur) un exemple de cette nécessaire réflexion conjointe.

La deuxième partie est constituée par deux contributions dues à des historiens, P. A. David et P. Temin ; elles se veulent des preuves par l'exemple. La première étudie les raisons historico-économiques du choix du système de lettres QWERTY sur les machines à écrire et la deuxième les difficultés institutionnelles récentes d'ATT pour la fixation de ses tarifs. Leur but est de montrer comment l'histoire est indispensable, d'une part, pour clarifier les relations entre théorie et observations et, d'autre part, pour expliquer certaines situations économiques actuelles qui échappent à la stricte rationalité théorique.

La troisième partie est formée par un ensemble de commentaires suscités par les contributions des deux premières parties. On notera, en particulier, celle de Donald McCloskey qui insiste sur l'identité des deux champs de recherche (p. 64) mais également, comme Solow, sur le caractère historique des lois économiques comme la loi de la demande. Or, si certains économistes peuvent admettre ce fait, bien peu sont prêts à en tenir réellement compte. Cette historicité des lois de l'économie est un aspect essentiel du débat pour beaucoup de commentateurs. W.W. Rostow incite les historiens à rejeter la théorie néo-classique et à jouer un rôle plus actif dans l'élaboration et la dynamisation des modèles proposés par les théoriciens. Beaucoup de difficultés de la théorie économique (emploi, technologie, développement) pourraient ainsi être résolues (p. 74). G. Wright considère de même que cette historicité des lois économiques justifierait un enseignement historique approfondi dans les cycles universitaires supérieurs. C. P. Kindleberger abonde dans le même sens. Dans la formation des étudiants, ce qu'il préfère appeler l'économie historique apporte une compréhension plus profonde des affaires du monde et surtout une intuition économique supérieure à beaucoup de théories ou de manipulations statistiques.

Cet ouvrage au ton très libre et à la lecture stimulante appelle trois remarques. En premier lieu, une certaine ambiguïté porte sur la notion d'histoire économique. Pour les chercheurs américains, il s'agit essentiellement d'histoire contemporaine (donc celle de sociétés capitalistes ou dominées par celles-ci) et souvent très formalisée (comme la New Economic History, très inspirée des 
modèles néo-classiques rejetés par W. W. Rostow). Ainsi tous les contributeurs, dont les historiens, enseignent dans le département économie de leur université. D'où la légitimité du thème de ce livre dans le contexte américain et la pertinence des critiques envers une histoire économique qui confond souvent emprunts et mimétisme. En second lieu, ce livre apparaît comme un produit typique du pragmatisme anglo-saxon, comme en témoigne la conception souvent naïve des apports mutuels des deux disciplines. Aucune tentative de théorisation sur les fondements épistémologiques des deux disciplines ou sur leurs implications idéologiques n'est esquissée. Le lecteur (européen !) reste insatisfait devant la seule évocation trop rapide du problème si essentiel de l'historicité des lois économiques alors que les anthropologues réfléchissent depuis si longtemps sur des thèmes analogues. En troisième lieu, nombre de contributions étonnent par leur caractère très critique envers l'économie théorique, surtout quand elles sont écrites par des théoriciens aussi remarquables que Arrow ou Solow. Ce trait témoigne d'une inquiétude qui traverse la pensée économique théorique actuelle qui doute non de sa technicité (jamais remise en cause en tant que telle dans cet ouvrage) mais de la validité de ses postulats et de la pertinence des résultats qu'ils induisent. Cette réflexion sur l'histoire économique traduit le même sentiment que celui qui justifie certaines publications épistémologiques actuelles. En ce sens, ce livre démontre peut-être l'existence d'une chance à saisir pour les historiens et les économistes qui veulent construire une science économique large et ouverte, analogue à celle que Solow invoque et qui ressemble par bien des aspects à celle que d'autres, comme le sociologue-économiste A.O. Hirschman, envisagent.

Jean-Yves GrenIER. 